\section{BMJ Open Respiratory Research}

\title{
Identifying causation in hypersensitivity pneumonitis: a British perspective
}

Christopher Michael Barber (D) , ${ }^{1}$ P Sherwood Burge, ${ }^{2}$ Jo R Feary, ${ }^{3}$ Helen Parfrey, ${ }^{4}$ Elizabeth A Renzoni, ${ }^{5}$ Lisa G Spencer, ${ }^{6}$ Gareth I Walters, ${ }^{2}$ Ruth E Wiggans, ${ }^{7}$ on behalf of the GB HP Survey Participants

\section{ABSTRACT}

Background Establishing whether patients are exposed to a 'known cause' is a key element in both the diagnostic assessment and the subsequent management of hypersensitivity pneumonitis (HP).

Objective This study surveyed British interstitial lung disease (ILD) specialists to document current practice and opinion in relation to establishing causation in HP. Methods British ILD consultants (pulmonologists) were invited by email to take part in a structured questionnaire survey, to provide estimates of demographic data relating to their service and to rate their level of agreement with a series of statements. A priori 'consensus agreement' was defined as at least $70 \%$ of participants replying that they 'Strongly agree' or 'Tend to agree'.

Results 54 consultants took part in the survey from 27 ILD multidisciplinary teams. Participants estimated that $20 \%$ of the patients in their ILD service have HP, and of these, a cause is identifiable in $32 \%$ of cases. For patients with confirmed HP, an estimated $40 \%$ have had a bronchoalveolar lavage for differential cell counts, and $10 \%$ a surgical biopsy. Consensus agreement was reached for 25 of 33 statements relating to causation and either the assessment of unexplained ILD or management of confirmed HP.

Conclusions This survey has demonstrated that although there is a degree of variation in the diagnostic approach for patients with suspected HP in Britain, there is consensus opinion for some key areas of practice. There are several factors in clinical practice that currently act as potential barriers to identifying the cause for British HP patients.

\section{INTRODUCTION}

Hypersensitivity pneumonitis (HP) is a common form of interstitial lung disease (ILD), with widely varying causes and clinical outcomes. ${ }^{1}$ Differentiating HP from other forms of fibrotic ILD is a common diagnostic dilemma for multidisciplinary teams (MDTs), ${ }^{2}$ resulting in a low level of diagnostic agreement internationally. ${ }^{3}$

Identifying exposure to a 'known cause' of HP remains a key element, both in the diagnostic approach for patients with unexplained ILD and in the management of confirmed

\section{Key messages}

Identifying causation in hypersensitivity pneumonitis is a common challenge for multidisciplinary teams, and there is little published research to inform the optimal approach.

- Interstitial lung disease specialists estimated that a cause is identifiable in around $32 \%$ of British HP cases, and agreed that there are several barriers that prevent this for most patients.

- This paper provides the first British data on causation in hypersensitivity pneumonitis and presents a series of agreed Consensus Statements for the investigation and management of these patients.

HP. ${ }^{4-8}$ Despite this, the optimal approach for establishing causation remains to be determined, ${ }^{9}$ with up to $63 \%$ of cases having no identifiable aetiological agent. ${ }^{10-17}$

The aim of this study was to document current practice and opinion among British ILD specialists (pulmonologists) in relation to establishing causation in HP.

\section{METHODS}

A structured questionnaire was developed by a Steering Committee $(n=8)$ of clinicians taken from recent or current members of the British Thoracic Society Specialist Advisory Groups for Interstitial or Occupational and Environmental Lung Disease with experience of managing HP. The series of statements for agreement were developed to gather expert opinion and identify areas of best practice at a national level.

Once the content of the questionnaire was agreed by the Steering Committee, the lead respiratory consultants of all British ILD MDTs were contacted via email and invited to participate in the 'Great British HP Survey'. Participants were asked to provide the email address of the other respiratory consultant members of their regional ILD MDT, and 
these individuals were also invited to participate by email. The number of participants was not restricted, and reminder emails were sent to improve participation. Data were collected between March and August 2018. Participants completed the questionnaire via a Web link (SurveyMonkey) provided in the email and were required to answer all questions. Responses required were predominantly numerical estimates or level of agreement/disagreement with statements devised by the Steering Committee (Strongly Agree, Tend to Agree, Neither Agree nor Disagree, Tend to Disagree and Strongly Disagree). A priori, 'Consensus Agreement' was defined as $70 \%$ or greater of participants replying that they 'Agree' (Strongly or Tend to). Participants were asked to rate how commonly their ILD service suspected a range of known domestic and occupational causes. For statements relating to causation, agreement that an exposure was a 'common cause' was defined as participants reporting that a cause was 'Commonest' or 'Common'. Median and IQR for numerical estimates were calculated using the online IQR calculator (EasyCalculation.com).

The full survey comprised 126 questions or statements covering background demographic information, causation, clinical assessment, prognosis and management. Where appropriate, participants were also given the opportunity to provide free text comments for certain questions. The results from the relevant sections of the survey relating to demographic data and causation are presented in this manuscript.

\section{Patient and public involvement}

This research was unfunded and was done without patient involvement. Patients were not invited to comment on the study design and were not consulted to develop patient relevant outcomes or interpret the results. Patients were not invited to contribute to the writing or editing of this document for readability or accuracy.

\section{RESULTS}

Fifty-four ILD specialist consultants (online supplementary appendix 1) took part in the survey (45 from England, 6 from Scotland and 3 from Wales) representing 27 ILD MDTs. Participants had worked in ILD MDTs for a median of 10 years (IQR 5-15 years), representing a cumulative total of 537 years of clinical practice. Thirty-five $(65 \%)$ reported that they have routine access to bronchoalveolar lavage (BAL) differential cell counts, and nine $(17 \%)$ routine access to cryobiopsy. Access to occupational lung disease (OLD) services varied between centres, with $39(72 \%)$ participants agreeing that they have an established route of referral to a regional OLD service, and $22(41 \%)$ agreeing that they work in an ILD MDT that has a consultant with expertise in OLD.

Participants estimated that 20\% (IQR 15\%-30\%) of the patients in their ILD service have HP as a final diagnosis, and of these, $40 \%$ (IQR 3\%-75\%) have had a BAL and $10 \%$ (IQR 5\%-20\%) a surgical biopsy. Thirty (56\%) of

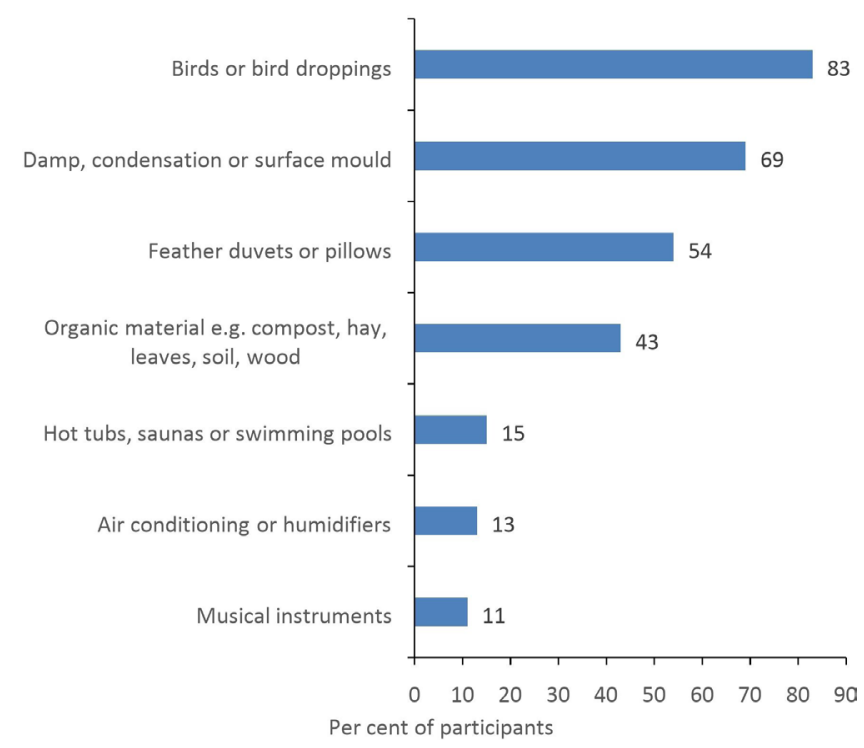

Figure 1 Commonly suspected domestic causes of HP $(n=54)$. HP, hypersensitivity pneumonitis.

those taking part in the survey stated that their preferred classification for HP is based on the predominant radiological feature on high resoltion CT scan (inflammatory, mixed or fibrotic HP), whereas nine (17\%) prefer classification based on duration of symptoms (acute, subacute or chronic HP).

Participants estimated that they are able to identify a cause in 32\% (IQR 20\%-50\%) of patients diagnosed with HP. Thirty-two $(59 \%)$ respondents agreed that in the ILD service they worked in, HP is commonly attributed to domestic exposures in the home or garden, whereas the corresponding figure for workplace exposures was only $21(39 \%)$. The most commonly suspected domestic and occupational causes, respectively, are exposure to avian proteins (figure 1) and metalworking fluids (MWFs)/ coolants (figure 2).

Twenty-five statements reached consensus agreement for either the assessment of patients with unexplained ILD or management of confirmed HP (tables 1 and 2, respectively). Eight statements did not reach consensus agreement (table 3 ).

\section{DISCUSSION}

This study presents the first British data relating to establishing HP causation, based on the opinion of ILD specialists with a cumulative total of over 500 years of clinical practice. There was a high level of consensus agreement among participants that identifying exposure to a known cause of HP is important, both for the assessment of patients with unexplained ILD and the management of patients with confirmed disease. There was also a clear consensus view that in many cases of HP in Britain it is not possible to identify a cause, and that there are identifiable barriers that contribute to this in day-to-day clinical practice. 
The main limitations of this study are twofold. First, as there is no national database for HP, the survey results are reliant on estimates from practicing clinicians, rather than routinely recorded British outcome data. The second consideration is that as the overall participation rate was $<50 \%$ of those invited by email, the results may not be truly representative of the full range of national practice and opinion.

Participants in the GB HP survey estimated that on average, 20\% of all ILD cases in their service have HP as a final diagnosis, a figure that is slightly higher than the $2 \%-15 \%$ range reported from ILD registries or database studies from other countries. ${ }^{15}{ }^{17-23}$ It is not possible to determine whether this difference is real, or due to the limitation of our study design. Participants also reported that on average, $40 \%$ of patients diagnosed with HP have had a BAL, although this estimate varied widely (between $0 \%$ and 100\%), suggesting that the British diagnostic approach varies markedly between centres.

British specialists were also asked to estimate what proportion of the patients diagnosed with HP, have an identifiable causative exposure. Although the median value for this estimate was $32 \%$, there was again a wide range of opinion (between $5 \%$ and $76 \%$ ). This degree of variability is perhaps not surprising, considering the lack of a standardised national approach to establishing HP causation. While the true epidemiology of HP in Britain remains unknown, the range of estimates from the GB HP survey were broadly consistent with the findings from other studies, where the proportion of HP patients without an identifiable cause was: $0 \%$ in $\operatorname{Japan}^{14} ; 4 \%$ in Brazil $^{15}$; $6 \%$ in Poland ${ }^{16}$; $28 \%$ in China ${ }^{17}$; and 25\%-63\% in the USA. ${ }^{10-13}$

Although consensus agreement was not reached, 54\% of participants agreed that they commonly attribute HP to be an 'idiopathic disease'. It is not possible from the survey results to determine whether this term is used to reflect the difficulties clinicians encounter in identifying the cause, or a true belief that HP can occur spontaneously (ie, without there being a cause to identify). Notably,

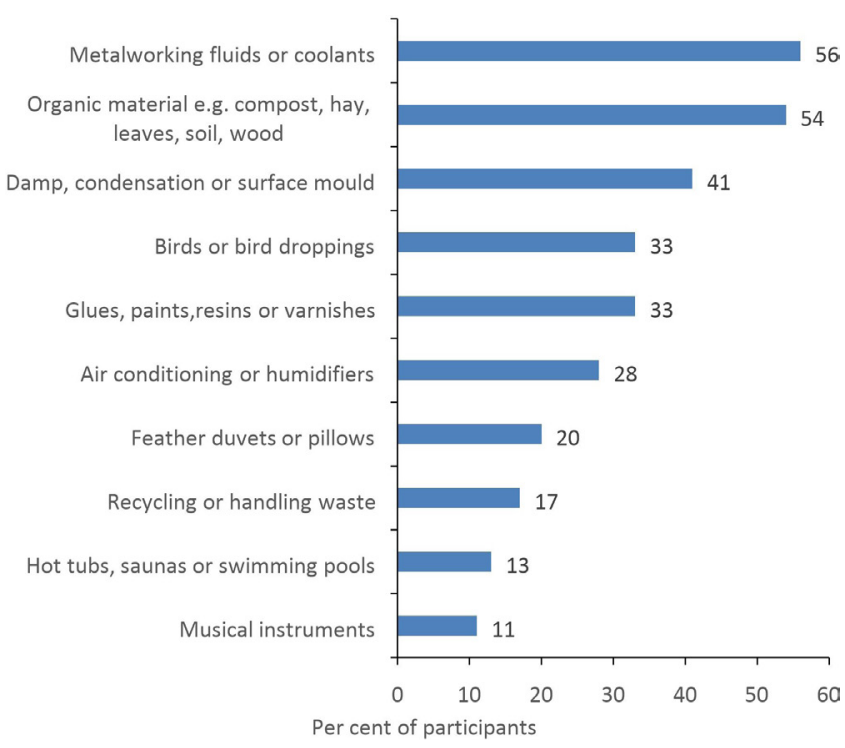

Figure 2 Commonly suspected occupational causes of HP ( $n=54)$. HP, hypersensitivity pneumonitis.

$43 \%$ of participants recognised that BAL lymphocytosis in HP can persist following cessation of exposure, and in some cases it may therefore be impossible to identify the cause, if it is no longer present in the work or home environment.

Causation in HP is likely to vary between countries due to a wide range of factors, including differences in geography, climate, housing and industry. In terms of identifying possible causes, GB HP survey participants reported that they more commonly attribute the disease to domestic exposures in the home or garden, than occupational exposures in the workplace. For domestic HP, the most commonly suspected exposures are to birds, bird droppings or feathers. This is in keeping with the majority of studies from other countries, where avian exposure has been the most commonly identified cause, accounting for $17 \%-66 \%$ of all cases. ${ }^{10-12} 14161724$ A recent retrospective single-centred US study also noted a high prevalence

Table 1 Agreed consensus statements for the assessment of HP diagnosis and/or cause in patients with unexplained ILD $(n=54)$

\section{Statements}

All patients with suspected HP on clinical or radiological grounds should be referred for a regional ILD MDT opinion. 83

In patients with ILD of unknown cause, the following clinical features increase the likelihood of a final diagnosis of HP:

- History of exposure to a known cause.

- Similar symptoms in cohabitants or coworkers.

Symptoms that improve away from the home or workplace.

The following tests should be requested for all patients with unexplained ILD:

Blood tests for specific IgG to known causes of HP.

In day-to-day practice, the following tests are useful in helping to differentiate HP from other forms of ILD:

- Specific lgG blood tests to known causes.

- Natural challenge (eg, improvement with avoidance of exposure).

HP, hypersensitivity pneumonitis; ILD, interstitial lung disease; MDT, multidisciplinary team. 
Table 2 Agreed consensus statements for the management of patients with confirmed HP $(n=54)$

\section{Statements}

The main aim of HP management is (where possible) to identify a cause and assist patients in avoiding further exposure.

A domestic cause of HP should be suspected if patients report that symptoms occur a few hours after a specific exposure in the home environment, or improve away from home, for example, following a 1- to 2-week holiday.

An occupational cause of HP should be suspected if patients report that symptoms occur a few hours after a specific exposure in the workplace, or improve away from work, for example, on rest days or holidays.

In many cases of confirmed HP it is difficult to identify a cause.

Identifying the cause of HP is difficult in some cases:

- Due to the limited range of specific IgG blood tests available.

- As there is no standardised facility to measure causative exposures in the home.

- As there is no standardised facility to measure causative exposures in the workplace.

As there is no standardised questionnaire to identify causes in the home.

- As there is no standardised questionnaire to identify causes in the workplace.

As there is no routinely available facility to carry out home or workplace visits.

Specific IgG titres to the cause (where available) may remain elevated in the blood following cessation of exposure 85 and are not a reliable method of identifying ongoing exposure.

Prognosis in HP:

- Is very variable.

- Is mainly related to the degree and pattern of fibrotic change at the time of diagnosis.

- Is worse for patients where an identified cause cannot be avoided.

- Is worse for patients where a cause cannot be identified.

The following features are associated with 'reversible disease', that is, the potential for some degree of clinical improvement with cessation of exposure and/or immunosuppression:

- Improvement of symptoms away from the home or workplace:

In a proportion of biopsy proven HP, fibrosis progresses despite cessation of exposure to the cause.

HP, hypersensitivity pneumonitis.

of contact with avian protein in biopsy proven chronic HP cases (29\% to bird and $58 \%$ to down), and incorporated a history of exposure into a prediction model. ${ }^{4} \mathrm{~A}$ slightly different pattern of causation for chronic fibrotic HP was reported from a retrospective single-centred Brazilian study, as exposure to mould (29\% of cases) was a more common cause than contact with birds/feathers (23\% of cases). ${ }^{15}$

Occupational exposures are also an important cause of $\mathrm{HP},{ }^{25}$ accounting for an estimated $19 \%$ of all cases..$^{26}$ The GB HP survey identified variable access to specialist OLD services nationally, with only $41 \%$ of participants reporting

Table 3 Statements not reaching consensus agreement for the assessment of HP diagnosis and/or cause in patients with unexplained ILD $(n=54)$

\section{Statements}

In day-to-day practice, the following tests are useful in helping to differentiate HP from other forms of ILD:

- Lymphocyte proliferation tests.

- Home or workplace visit.

Specific inhalation challenge (hospital based).

In patients where HP is the first choice clinical and radiological diagnosis, a BAL differential cell count:

$\checkmark$ Is not required if there is a clear history of exposure.

- Is not required if there is a clear history of exposure and an elevated level of specific IgG to a known cause.

Normal levels of specific lgG to avian proteins effectively exclude bird or feather down/duvet exposures as the cause of HP in exposed individuals.

BAL lymphocytosis may persist following cessation of exposure and is not a reliable method of identifying ongoing exposure.

BAL, bronchoalveolar lavage; HP, hypersensitivity pneumonitis; ILD, interstitial lung disease. 
that their ILD MDT has a consultant with expertise in OLD, and $72 \%$ an established route of referral. For occupational causes, the most commonly suspected exposures are to MWF or organic material (eg, compost, hay, leaves, soil, wood). This is entirely in keeping with data from the UK reporting scheme for occupational HP between 1996 and 2015, where exposure to MWF (35\% of cases) and farming ( $17 \%$ of cases) were the most common causes. ${ }^{27}$ The reporting data demonstrated that over this time period, 'Metalworking fluid HP' has become the most commonly reported cause of occupational HP in the UK, a change in epidemiology that merits further research. ${ }^{28}$

Given the poor level of diagnostic agreement for HP between MDTs, the GB HP survey selected a relatively low $(70 \%)$ level for consensus agreement. ${ }^{729}$ Utilising this threshold, the survey found consensus for 25 of 33 statements relating to different aspects of HP and causation, with many having much higher levels of agreement. Although methodological differences do not allow exact comparisons, the results from an international Delphi for HP diagnosis offer a valuable comparator for some of the British consensus statements. Of note, the international Delphi comprised three rounds, required $80 \%$ agreement for consensus, and sought views from 45 experts from 14 countries ( 22 from North America and 3 from the UK). ${ }^{7}$ Despite these differences, the international Delphi and GB HP surveys found similar consensus agreement for the importance of certain aspects of HP diagnosis, including MDT case discussion, identifying exposure to a known cause and recognising a temporal relation between symptoms and exposure. Another common view shared by participants of the two studies related to the use of specific inhalation challenges (SIC) to confirm causation in HP. Although these are routinely carried out as part of the diagnostic approach in some centres, ${ }^{8} \mathrm{SIC}$ was only rated as diagnostically useful by $35 \%$ of British ILD specialists and as diagnostically important by $42 \%$ in the first round of the international Delphi.

One notable difference between the findings of the two studies related to the utility of specific IgG blood testing to known causes of HP. The diagnostic value of specific IgG testing did not reach consensus for importance in the international Delphi, but in the first round, the majority (53\%) rated it as important. ${ }^{7}$ In contrast, the GB HP survey participants did reach consensus agreement that specific IgG blood tests: are useful in helping to differentiate HP from other forms of ILD; should be requested for all patients with unexplained ILD; and act as a barrier to establishing causation in some cases due to the limited panel available. The diagnostic importance of IgG testing was further highlighted in the survey as the majority of British ILD clinicians $(61 \%)$ agreed that in the context of a clear exposure history, typical radiology and an elevated level of specific IgG to a known cause, a BAL differential cell count is not required.

There was also consensus agreement among British ILD specialists that the lack of routine provision for home/workplace visits acts as a barrier to identifying the cause of HP in some cases. In Britain, ILD MDTs operate within a government funded National Health Service, and there is no standardised approach for investigating possible causes of HP, and no routinely available funding for home/workplace visits for environmental sampling. The current approach to identifying HP causation in Britain is therefore often heavily reliant on patient history and the results of specific IgG testing. Alternative strategies to antigen identification have been suggested in other countries, based either on the results of bespoke IgG testing or SIC to extracts of microorganisms cultured directly from the home or workplace. ${ }^{3031}$ While this type of approach is promising, it requires further validation, and is not likely to impact on HP practice in Britain in the near future.

In conclusion, the GB HP survey has demonstrated national variation in the utilisation of invasive diagnostic tests in HP, but consensus opinion for some of the key aspects of practice relating to establishing causation. The survey has highlighted that ILD specialists believe this to be an important area of practice, affecting clinical outcomes, but that there are identifiable barriers preventing this for most British HP patients.

\section{Author affiliations}

${ }^{1}$ Centre for Workplace Health, Sheffield Teaching Hospitals NHS Foundation Trust, Sheffield, UK

${ }^{2}$ Birmingham Regional NHS Occupational Lung Disease Service, Birmingham Chest Clinic, Birmingham, UK

${ }^{3}$ Department of Occupational Lung Disease, Royal Brompton and Harefield NHS Foundation Trust, London, UK

${ }^{4}$ Cambridge ILD Service, Royal Papworth Hospital NHSFT, Cambridge, UK

${ }^{5}$ Interstitial Lung Disease Unit, Royal Brompton and Harefield NHS Foundation Trust, London, UK

${ }^{6}$ Liverpool Interstitial Lung Disease Service, University Hospital Aintree,

Liverpool, UK

${ }^{7}$ Chest Clinic, Wythenshawe Hospital, Manchester, UK

Acknowledgements The authors are grateful to the GB HP survey participants for taking time to complete the survey.

Collaborators Huzaifa Adamali, Suresh Babu, Shaney Barrat, Alexander Basran, Paul Beirne, Stephen Bianchi, George Chalmers, Nazia Chaudhuri, Sarah Davies, Owen Dempsey, Sinan Eccles, Christine Fiddler, Noleen Foley, lan Forrest, Sophie Fletcher, Peter George, Salman Ghani, Michael Gibbons, Mike Greenstone, Simon Hart, Nick Hirani, Jennifer Hoyle, Rachel Hoyles, John Hutchinson, Gisli Jenkins, Eoin Judge, Ajay Kamath, Maria Kokosi, Candy Lee, Toby Maher, Ben Marshall, Neil McAndrew, Philip Molyneux, Douglas Morrison, Steve 0'Hickey, Joanna Porter, Steve Renshaw, Charles Sharp, Nicky Simler, Mark Spears, Alexander Spiers, Katherine Spinks, Monica Spiteri, Chris Stenton, Sharon Sturney, Chris Warburton, Sarah Wiscombe, Felix Woodhead.

Contributors CMB planned the study, conducted the survey, drafted the manuscript and is responsible for guaranteeing the overall content of the research. $\mathrm{SB}, \mathrm{JRF}, \mathrm{HP}, \mathrm{EAR}, \mathrm{LGS}, \mathrm{GIW}$ and REW contributed to designing the content of the survey, interpreting the results and had input into the writing of the final manuscript.

Funding The authors have not declared a specific grant for this research from any funding agency in the public, commercial or not-for-profit sectors.

Competing interests None declared.

Patient consent for publication Not required.

Provenance and peer review Not commissioned; externally peer reviewed.

Data availability statement All data relevant to the study are included in the article or uploaded as supplementary information.

Open access This is an open access article distributed in accordance with the Creative Commons Attribution Non Commercial (CC BY-NC 4.0) license, which 
permits others to distribute, remix, adapt, build upon this work non-commercially, and license their derivative works on different terms, provided the original work is properly cited, appropriate credit is given, any changes made indicated, and the use is non-commercial. See: http://creativecommons.org/licenses/by-nc/4.0/.

ORCID iD

Christopher Michael Barber http://orcid.org/0000-0003-2234-4165

\section{REFERENCES}

1 Kouranos V, Jacob J, Nicholson A, et al. Fibrotic hypersensitivity pneumonitis: key issues in diagnosis and management. J Clin Med 2017;6.

$2 \mathrm{HE}$ J, Corte TJ, Moodley Y, et al. Evaluating the interstitial lung disease multidisciplinary meeting: a survey of expert centres. BMC Pulm Med 2016;16.

3 Walsh SLF, Wells AU, Desai SR, et al. Multicentre evaluation of multidisciplinary team meeting agreement on diagnosis in diffuse parenchymal lung disease: a case-cohort study. Lancet Respir Med 2016;4:557-65.

4 Johannson KA, Elicker BM, Vittinghoff E, et al. A diagnostic model for chronic hypersensitivity pneumonitis. Thorax 2016;71:951-4.

5 Vasakova M, Morell F, Walsh S, et al. Hypersensitivity pneumonitis: perspectives in diagnosis and management. Am J Respir Crit Care Med 2017;196:680-9.

6 Salisbury ML, Myers JL, Belloli EA, et al. Diagnosis and treatment of fibrotic hypersensitivity pneumonia. where we stand and where we need to go. Am J Respir Crit Care Med 2017;196:690-9.

7 Morisset J, Johannson KA, Jones KD, et al. Identification of diagnostic criteria for chronic hypersensitivity pneumonitis: an international modified Delphi survey. Am J Respir Crit Care Med 2018;197:1036-44.

8 Morell F, Ojanguren I, Cruz MJ, et al. Hypersensitivity pneumonitis. toward a less invasive diagnostic procedure. Archivos de Bronconeumología 2018;54:445-6.

9 Molyneaux PL, Maher TM. Time for an international consensus on hypersensitivity pneumonitis. A call to arms. Am J Respir Crit Care Med 2017;196:665-6.

10 Hanak V, Golbin JM, Ryu JH. Causes and presenting features in 85 consecutive patients with hypersensitivity pneumonitis. Mayo Clin Proc 2007;82:812-6.

11 Mooney JJ, Elicker BM, Urbania TH, et al. Radiographic fibrosis score predicts survival in hypersensitivity pneumonitis. Chest 2013;144:586-92.

12 Fernández Pérez ER, Swigris JJ, Forssén AV, et al. Identifying an inciting antigen is associated with improved survival in patients with chronic hypersensitivity pneumonitis. Chest 2013;144:1644-51.

13 Adegunsoye A, Oldham JM, Fernández Pérez ER, et al. Outcomes of immunosuppressive therapy in chronic hypersensitivity pneumonitis. ERJ Open Res 2017;3. doi:10.1183/23120541.00016-2017. [Epub ahead of print: 1708 2017]

14 Dobashi K, Akiyama K, Usami A, et al. Committee for Japanese guideline for diagnosis and management of occupational allergic diseases; Japanese Society of Allergology. Japanese guideline for occupational allergic diseases 2014. Allergol Int 2014;63:421-42.

15 Gimenez A, Storrer K, Kuranishi L, et al. Change in FVC and survival in chronic fibrotic hypersensitivity pneumonitis. Thorax 2018;73:391-2.

16 Szturmowicz M, Barańska I, Jeedrych ME, et al. Hypersensitivity pneumonitis recognised in a single pulmonary unit, between 2005 and 2015 - comparison with recently proposed diagnostic criteria. Adv Respir Med 2019;87:83-9.

17 Wang LJ, Cai HR, Xiao YL, et al. Clinical characteristics and outcomes of hypersensitivity pneumonitis: a population-based study in China. Chin Med J 2019;132:1283-92.

18 Thomeer MJ, Costabe U, Rizzato G, et al. Comparison of registries of interstitial lung diseases in three European countries. Eur Respir $J$ Supp/ 2001;32:114s-8.

19 Xaubet A, Ancochea J, Morell F, et al. Spanish group on interstitial lung diseases, SEPAR. Report on the incidence of interstitial lung diseases in Spain. Sarcoidosis Vasc Diffuse Lung Dis 2004;21:64-70.

20 Kornum JB, Christensen S, Grijota M, et al. The incidence of interstitial lung disease 1995-2005: a Danish nationwide populationbased study. BMC Pulm Med 2008;8:24

21 Karakatsani A, Papakosta D, Rapti A, et al. Hellenic interstitial lung diseases group. epidemiology of interstitial lung diseases in Greece. Respir Med 2009;103:1122-9.

22 Kundu S, Mitra S, Ganguly J, et al. Spectrum of diffuse parenchymal lung diseases with special reference to idiopathic pulmonary fibrosis and connective tissue disease: an eastern India experience. Lung India 2014;31:354-60.

23 Hyldgaard C, Hilberg O, Muller A, et al. A cohort study of interstitial lung diseases in central Denmark. Respir Med 2014;108:793-9.

24 Lacasse Y, Selman M, Costabel U, et al. Clinical diagnosis of hypersensitivity pneumonitis. Am J Respir Crit Care Med 2003;168:952-8.

25 Quirce S, Vandenplas O, Campo P, et al. Occupational hypersensitivity pneumonitis: an EAACI position paper. Allergy 2016;71:765-79.

26 Blanc PD, Annesi-Maesano I, Balmes JR, et al. The occupational burden of nonmalignant respiratory diseases. An official American thoracic Society and European respiratory Society statement. Am J Respir Crit Care Med 2019;199:1312-34.

27 Barber CM, Wiggans RE, Carder M, et al. Epidemiology of occupational hypersensitivity pneumonitis; reports from the SWORD scheme in the UK from 1996 to 2015. Occup Environ Med 2017;74:528-30.

28 James PL, Cannon J, Barber CM, et al. Metal worker's lung: spatia association with Mycobacterium avium. Thorax 2018;73:151-6.

29 Schoenberg NC, Barker AF, Bernardo J, et al. A comparative analysis of pulmonary and critical care medicine Guideline development methodologies. Am J Respir Crit Care Med 2017;196:621-7.

30 Millerick-May ML, Mulks MH, Gerlach J, et al. Hypersensitivity pneumonitis and antigen identification - an alternate approach. Respir Med 2016:112:97-105.

31 Bellanger A-P, Reboux G, Rouzet A, et al. Hypersensitivity pneumonitis: a new strategy for serodiagnosis and environmental surveys. Respir Med 2019;150:101-6. 